\title{
CONGENITAL BICUSPID AORTIC VALVE - A CASE REPORT
}

\author{
AMIN MR ${ }^{1}$, HASAN MN ${ }^{2}$, BEGUM M$^{3}$, MEHEDI MT ${ }^{4}$
}

\begin{abstract}
:
Among all the congenital heart disease, bicuspid aortic valves (BAVs) are cardiac valvular anomaly, occurring in 1-2\% of the general population. It is twice as common in males as in females. In many cases, a bicuspid aortic valve will cause no problems. However, BAV may become calcified later in life, which may lead to varying degrees of severity of aortic stenosis that will manifest as murmurs. If the leaflets do not close correctly, aortic regurgitation can occur. These mixed clinical finding sometimes confuses physician and culminating to considering other differentials, needs further more subsequent investigation and imaging, thus prolonging evaluation time and delaying decision making for treatment plan.
\end{abstract}

Key words: Bicuspid aortic valve, aortic stenosis, aortic regurgitation.

J Dhaka Med Coll. 2014; 23(1) : 128-130.

\section{Introduction:}

The aortic valve is a one-way valve between the heart and the aorta. Normally, the aortic valve has three small flaps or leaflets that open widely and close securely to regulate blood flow, allowing blood to flow from the heart to the aorta and preventing blood from flowing backwards into the heart. In bicuspid aortic valve disease (BAVD), the valve has only two leaflets. With this deformity, the valve doesn't function perfectly ${ }^{1}$. The actual cause of bicuspid aortic valve disease is not completely clear. Bicuspid aortic valve is an inheritable condition, with a demonstrated association with Notch $1^{2}$. Its heritability is as high as $89 \%$-.Both familial clustering and isolated valve defects have been documented. The incidence of bicuspid aortic valve can be as high as $10 \%$ in families affected with the valve problem ${ }^{3}$. Recent studies suggest that BAV is an autosomal dominant condition with incomplete penetrance. Other congenital heart defects are associated with bicuspid aortic valve at various frequencies, including coarctation of the aorta. Although bicuspid aortic valve disease is present at birth, it usually is not diagnosed until adulthood because the defective valve can function for years without causing symptoms. Rarely, the disease is so severe at birth that the baby develops congestive heart failure early in life. Calcium deposits on and around the leaflets eventually cause the valve to stiffen and narrow, developing aortic stenosis.

\section{Case Report:}

A 23 years old housewife, admitted in BSMMU complaining of exertional fatigability for last 1 year and he feels fatigueness and mild shortness of breath on moderate exertion which are relieved on taking rest. These are not associated with any chest pain, palpitation, syncope, skin pigmentation or alteration of bowel habit. She denies any history of Bronchial Asthma and rheumatic fever in her childhood. She is married for three years . She had an one years old child and complained of exertional shortness of breath during her last trimester.

She is of average body built and nutrition, decubitus on choice, pulse rate was 78 beats/

1. Dr. Md. Rasul Amin, Medical Officer, Department of Cardiology, Bangabandhu Sheikh Mujib Medical University (BSMMU), Dhaka.

2. Dr. Md. Nazmul Hasan, Resident, Department of Cardiology, Bangabandhu Sheikh Mujib Medical University (BSMMU), Dhaka.

3. Dr. Masuma Begum, Associate Professor, Department of Community Medicine, Holy Family Red Crescent Medical College, Dhaka.

4. Dr. Md Tarique Mehedi, Deputy Director \& In-charge, Red Crescent Blood Center, Dhaka.

Correspondence: Dr. Md Nazmul Hasan, Resident, Department of Cardiology, Bangabandhu Sheikh Mujib Medical University (BSMMU), Dhaka. Cell Phone: +8801675755638, Email: nazmulhasan45cmc@gmail.com 
minute, regular and of normal volume, Blood Pressure was 100/70 mmHg, JVP was normal. There is no anaemia, jaundice or skin pigmentation. Examination of the Precordium reveals there is a visible impulse in apical area, apex is in the left $5^{\text {th }}$ intercostal space just lateral to the midclavicular line which is forceful. There is a systolic thrill in the aortic area with an ejection systolic murmur, radiates to neck, left sternal edge and to the apex, the intensity of which increasing with leaning forwards and breath hold after expiration. There is another murmur, early diastolic in character, intensity $3 / 6$ radiates also to the left edge of the sternum and apex. Both of the lung fields are clear.

Among the investigations, 12 lead electrocardiogram shows left ventricular hypertrophy, X-ray chest denotes cardiomegaly. All others hematological and biochemical parameter are normal. Color Doppler echocardiogram suggesting of congenital aortic valvular heart disease in the form of bicuspid valve, it shows severe bicuspid aortic stenosis (PPG 139 mmHg, mean gradient $66.8 \mathrm{mmHg}$ ). There is a single diastolic closure line that is located eccentrically. Aortic valve cusps are thickened, calcified and restricted in lateral mobility. There is a post stenotic dilatation of ascending aorta about $38 \mathrm{~mm}$. There is diminished cusp separation and valve orifice has a fish mouth shape in systole. There is grade III aortic regurgitation with concentric left ventricular hypertrophy.

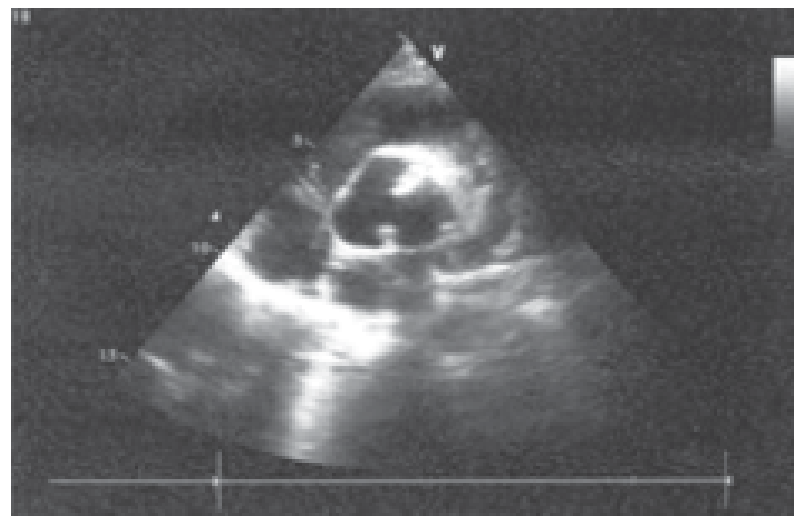

Fig.-1: Transthoracic echocardiogram shows single diastolic closure line with two cusps.

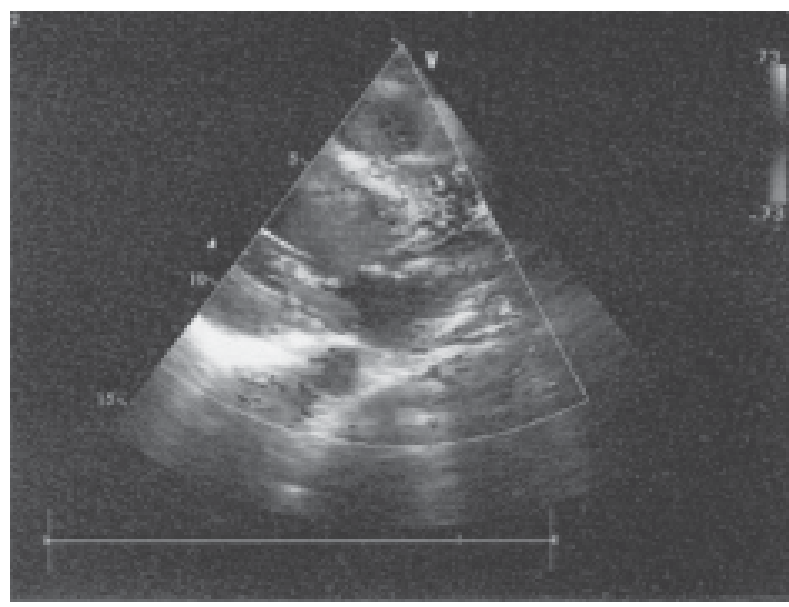

Fig.-2: Transthoracic echocardiogram (color flow) shows aortic regurgitation.

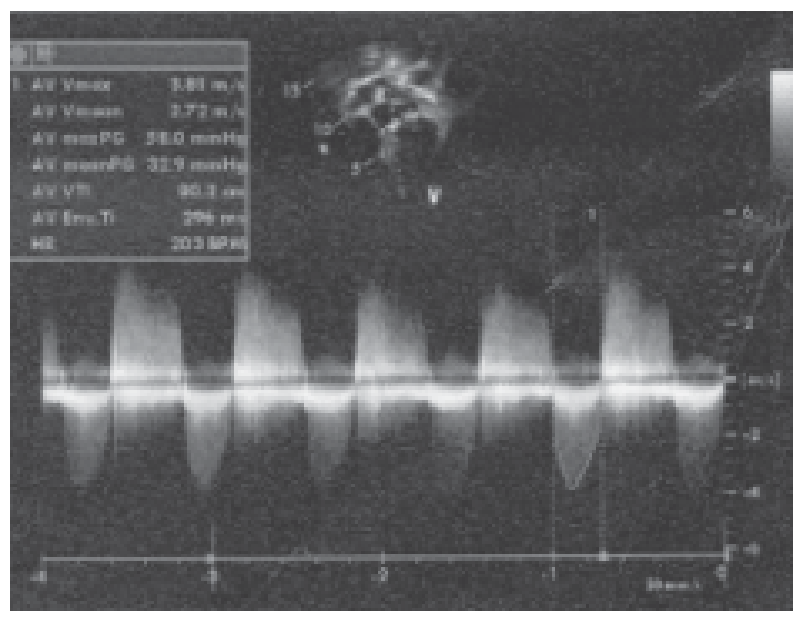

Fig.-3: Transthoracic echocardiogram (spectral flow) shows Aortic stenosis with regurgitation.

\section{Discussion:}

Symptoms of a stenotic valve include chest pain, shortness of breath and dizziness or fainting caused by inadequate blood flow to the brain. In this condition, a low volume pulse, narrow pulse pressure, a soft second heart sound with an ejection systolic murmur may be found with or without thrill. If the bicuspid valve does not close completely, blood can flow backwards into the heart developing aortic valve insufficiency or regurgitation, causing strain on the heart's lower left chamber, the left ventricle. Over time, the ventricle will dilate. The main symptom of aortic valve regurgitation is shortness of breath during exertion, like walking up stairs and an early diastolic murmur, high volume peripheral 
pulse and wide pulse pressure are the characteristic clinical findings. As the disease progresses, these symptoms start occurring more frequently, even without exerciset. The diagnosis can done by proper history, characteristic clinical findings and assisted with echocardiography or magnetic resonance imaging (MRI). Evidence of left ventricular hypertrophy may be determined by electrocardiogram. For diagnosed patients, genetic testing is done to allow for future offspring with the disease to be monitored and treated early in lifeu. In the most severe cases, when symptoms are present at birth or in early infancy, surgical repair of the valve must be performed immediately. In other cases, people can go their whole lives without knowing they have BAVD. About $80 \%$ of people with BAVD will require surgical treatment to repair or replace the valve and part of the aorta, usually when they are in their 30 s or $40 \mathrm{sv}$. Patients with bicuspid aortic valve should be followed by a cardiologist or cardiac surgeon. If the valve is normally functioning or minimally dysfunctional, average lifespan is similar to that of those without the anomaly.

\section{Conclusion:}

The tissue abnormality in patients with a bicuspid aortic valve is not confined to the valve leaflets; these patients are at increased risk of aortic aneurysm and dissection. At the tissue level, the aorta shows cystic medial necrosis, loss of elastic fibers, increased apoptosis, and altered smooth muscle cell alignment. When compared with patients with a trileaflet valve, patients with a bicuspid valve have larger aortic root dimensions and an increased rate of aortic dilation over time, with the degree of aortic dilation independent of valve hemodynamics. Given the increased risk of identifying a bicuspid aortic valve in firstdegree relatives having the same diagnosis, screening of this at-risk population should be considered. Echocardiographers should take particular care to identify bicuspid aortic valves in young patients because of the important long-term clinical consequences of this conditiont .

\section{References}

1. Garg V, Muth AN, Ransom JF et al. Mutations in NOTCH1 cause aortic valve disease. Nature 23005; 437 (7056): 270-4.

2. Otto CM, Bonow RO. Valvular Heart Disease. In: Bonow RO, Mann DL, Zipes DP, Libby P, eds. Braunwald's heart disease: a textbook of cardiovascular medicine. $9^{\text {th }}$ ed. Philadelphia: Saunders Elsevier; 2011.

3. Cripe L, Andelfinger G, Martin LJ, Shooner K, Benson DW. Bicuspid aortic valve is heritable. J Am Coll Cardiol 2004; 44 (1): 138-43.

4. Michelena HI, Desjardins VA, Avierinos JF et al. Natural history of asymptomatic patients with normally functioning or minimally dysfunctional bicuspid aortic valve in the community. Circulation 2008; $117(21)$ : 2776-2784.

5. Rison SC, Locke TP, Rosenthal E, Gandhi S. A man with hypertension and two murmurs. BMJ (Clinical Research) 2012; 344: e956.

6. Svensson LG. Aortic valve stenosis and regurgitation: an overview of management. J Cardiovasc Surg (Torino). 2008; 49(2): 297-303. 